\title{
Quantitative chemical proteomics reveals a Plk1 inhibitor-compromised cell death pathway in human cells
}

Cell Research (2014) 24:1141-1145. doi:10.1038/cr.2014.86; published online 1 July 2014

\section{Dear Editor,}

Because multiple cancers require Polo-like kinase (Plk) 1 for survival [1-3], Plk1 has been investigated intensively as a target for novel anti-cancer agents [46]. Although many small molecule Plk1 inhibitors have reached the clinic, our knowledge about their target profiles is limited, because their biochemical properties are determined mostly using in vitro kinase assays. It remains unclear whether this approach reflects the true pharmacodynamic properties of Plk1 inhibitors, since these assays often address only small portions of the human kinome and typically use a recombinant kinase and an artificial substrate. In the present study, for a more comprehensive characterization of the clinical Plk1 inhibitor BI2536, we treated HeLa cells with serial dilutions of this inhibitor.

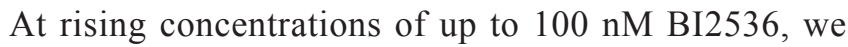
observed a significant mitotic arrest, a high percentage of rounded cells and increasing levels of cell death (Figure 1A and Supplementary information, Figure S1A). Above this drug concentration, the apoptotic activity did not further increase significantly or decreased to a small extend (Figure 1A and Supplementary information, Figure S1A), accompanied by a slight recovery of cell growth (Figure 1A and Supplementary information, Figure S1A, upper panels) and flattening of the cells as seen by microscopical inspection. This observation indicates that the drug response is biphasic and concentration-dependent with a small reduction of apoptosis at high doses compared to concentrations lower than $100 \mathrm{nM}$.

Since the sole inhibition of Plk1 could not account for the improved cell survival at elevated inhibitor concentrations, we investigated whether hitherto unknown targets of BI2536 could contribute to the unexpected apoptotic behavior of cancer cells. To this end, a Kinobead competition assay was applied to the quantitative profiling of the Plk1 inhibitor BI2536 [7, 8]. Kinobeads captured approximately two-thirds of the expressed kinome (Supplementary information, Figure S1B). Our experiment revealed a very narrow target profile for BI2536: six proteins exhibited $>33 \%$ binding reduction on Kinobeads at $250 \mathrm{nM}$ in the lysates (Supplementary information, Table S1 and Figure S1C). Among these newly identified targets, those commercially available kinase proteins were further analyzed in in vitro kinase assays: While at $1 \mathrm{nM}, \mathrm{BI} 2536$ interferes with the activity of CDC-like kinase 2 (CLK2) moderately, it is a potent inhibitor of Plk1, death-associated protein kinase 2 (DAPK-2) and calcium/calmodulin-dependent protein kinase kinase 2 (CAMKK2), confirming our results obtained from quantitative mass spectrometry (Figure 1B and Supplementary information, Figure S1B and S1C). Although Kinobeads affinity captures some 300 human kinases [7], there are 'blind spots' due to the lack of sufficient affinity of the immobilized ligands towards a particular kinase, or the low expression level of a certain kinase, preventing detection by mass spectrometry. Consequently, Plks are very often not detected using Kinobeads.

DAPKs constitute a family of Ser/Thr kinases that share cell death-related functions and have significant sequence homology within their kinase domains [9]. Hence, we tested the effect of BI2536 also on the activity of DAPK, the best-characterized member of the DAPK family, and observed that BI2536 is efficient in inhibiting DAPK activity in vitro (Figure 1B). To examine the direct binding of BI2536 to DAPK, we performed a displacement assay using the fluorescent ATP analog 2',3'-O-(2,4,6-trinitrophenyl) adenosine 5'-triphosphate (TNP-ATP) $[10,11]$. While TNP-ATP displays minimal fluorescence in solution, upon binding to a protein, a significant increase of fluorescence occurs. A reduction in fluorescence was observed upon addition of BI2536 for Plk1 and DAPK in a concentration-dependent manner suggesting the displacement of TNP-ATP from the ATPbinding sites (Figure 1C).

Since Plk1 inhibition leads to mitotic arrest in cancer cells followed by an induction of apoptosis, we investigated whether DAPK activity is regulated during cell cycle progression and might contribute to cell death induced by Plk1 inhibition. To follow the course of DAPK expression, cells treated with a Plk1-mismatch (MM) siRNA or a Plk1-specific siRNA were synchronized by 
A
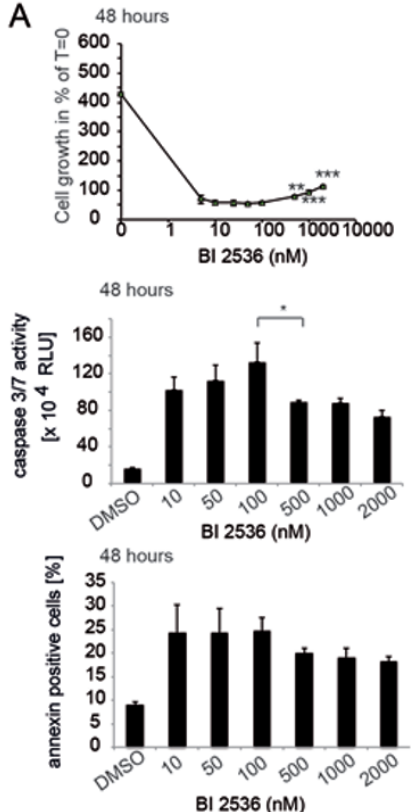

E Plk1 (10 nM)

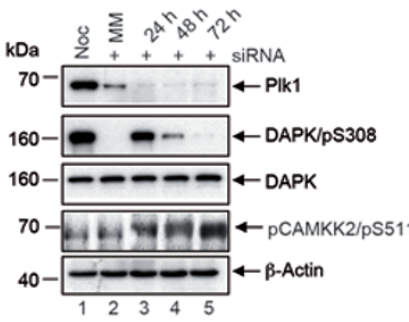

G

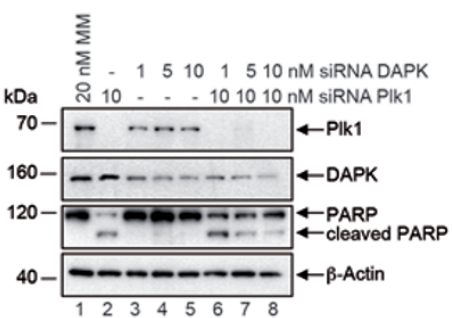

B

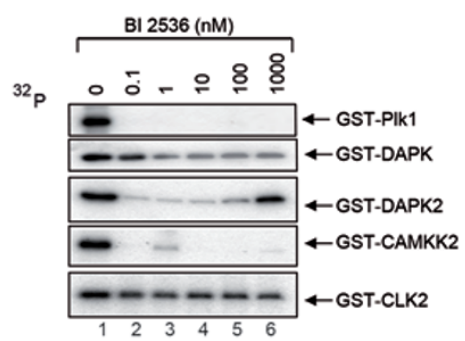

C

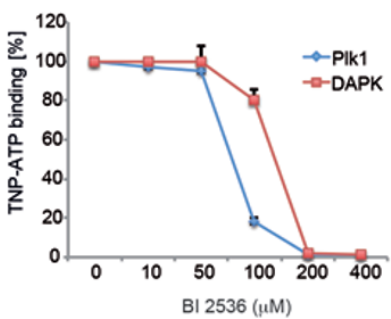

D
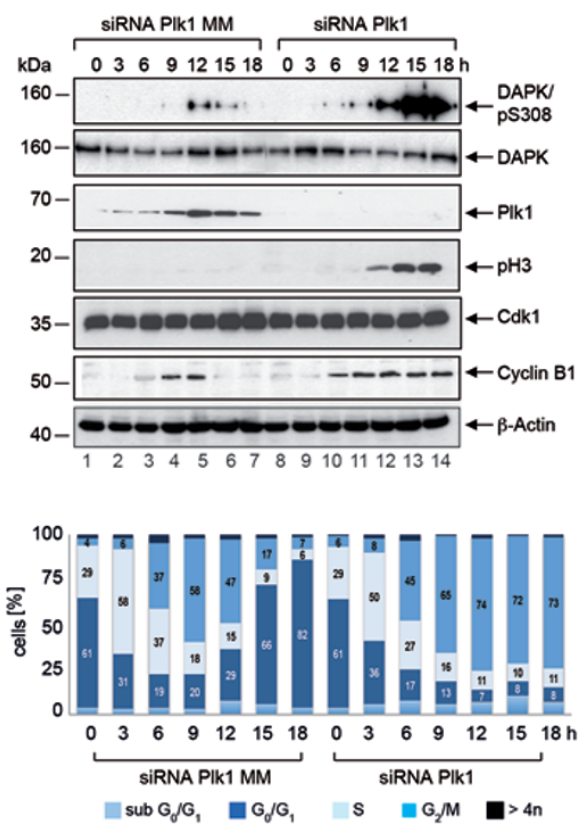

F
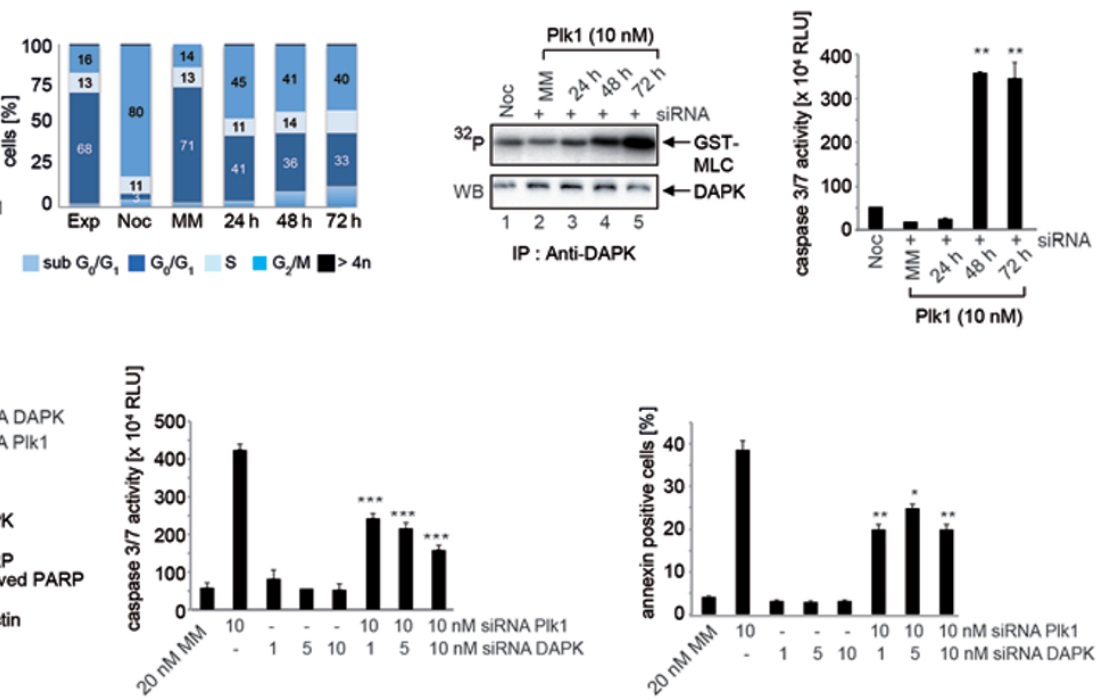

$\mathrm{H}$

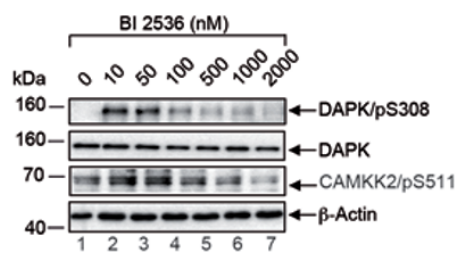

I

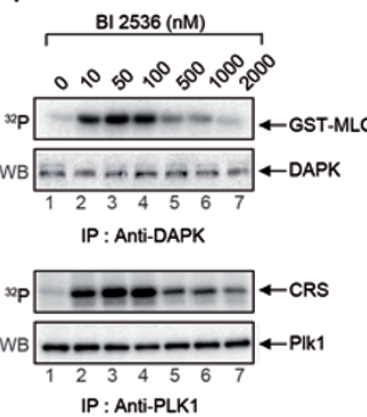

J
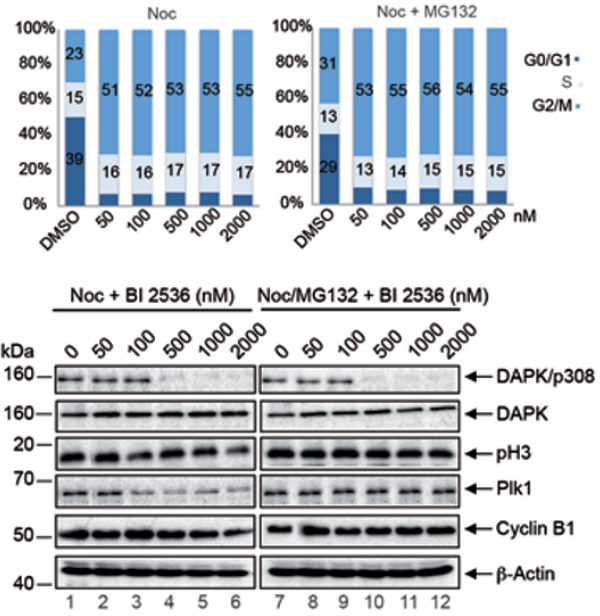
Figure 1 Characterization of the mitotic roles of DAPK in Plk1-inihibited HeLa cells. (A) Concentration-dependent proliferative and apoptotic responses upon BI2536 treatment. (B) Evaluation of novel BI2536 targets using in vitro kinase assays. The purified proteins were subjected to kinase assays and the autophosphorylation activities were monitored using $\gamma$-32P ATP. (C) TNP-ATP displacement assay for the analysis of BI2536 binding to Plk1 and DAPK. (D) Cells treated with Plk1 siRNA or Plk1-MM siRNA were arrested in G1/ $S$ by double thymidine-treatment. At the indicated time points, cells were lysed and immunoblotted for DAPK/pS308, DAPK, Plk1, pH3, Cdk1, Cyclin B1 and $\beta$-Actin (upper panel). Cell cycle was analyzed by FACS at the indicated time points (lower panel). (E) Cells were treated with nocodazole for $16 \mathrm{~h}$ or transfected with Plk1-MM siRNA as control for $24 \mathrm{~h}$ or Plk1 siRNA for $24 \mathrm{~h}, 48 \mathrm{~h}$ and $72 \mathrm{~h}$. Lysates were immunoblotted for PIk1, DAPK/pS308, DAPK, CAMKK2/pS511 and $\beta$-Actin (left panel). Cells were analyzed by FACS at the indicated time points (right panel). (F) DAPK immunoprecipitated from the lysates of siRNA-treated cells was subjected to kinase assays using GST-MLC as the substrate (left panel) and caspase-3/7 activity was determined (Caspase-Glo 3/7 Assay) (right panel). ${ }^{*} P<0.01$, Student's $t$-test, unpaired and two-tailed. (G) HeLa cells were transfected with siRNA as indicated. Twenty-four hours after transfection, cell lysates were analyzed by immunoblotting for Plk1, DAPK, PARP and $\beta$-Actin (left panel). Caspase-3/7 activity was determined (middle panel) and 7-AAD was used in conjunction with annexin $V$ staining to discriminate among the viable, apoptotic and necrotic cells using dual parameter FACS analysis (right panel). ${ }^{*} P<0.05,{ }^{* *} P<0.01,{ }^{* * *} P<0.001$, Student's t-test, unpaired and two-tailed. (H) Lysates of cells treated with increasing concentrations of BI2536 for $24 \mathrm{~h}$ were immunoblotted for DAPK/pS308, DAPK, CAMKK2/ pS511 and $\beta$-Actin. (I) DAPK and PIk1 immunoprecipitated from lysates were subjected to kinase assays using GST-MLC and cytoplasmic retention signal (CRS) of cyclin B1 as the substrates, respectively. (J) Cells enriched in mitosis by nocodazole- or nocodazole/ MG132-treatment were incubated with increasing concentrations of BI2536 for $24 \mathrm{~h}$. Cell cycle analyses by FACS (upper panel) and immunoblotting (lower panel) were then performed.

double-thymidine treatment and analyzed. The DAPK protein was present throughout the cell cycle (Figure 1D). Previously, it was reported that DAPK is autophosphorylated at S308, which correlates inversely with the ability of DAPK to phosphorylate its substrates [12]. Dephosphorylation of S308 strongly enhances the death promoting activity of DAP kinase [13]. While synchronized cells treated with Plk1-MM siRNA showed a transient signal for DAPK/pS308, Plk1-depleted cells that were arrested in mitosis with high levels of cyclin B1 and pH3 showed very strong signals for DAPK/pS308 (Figure 1D), suggesting that the activity of DAPK is autoinhibited during mitosis. Furthermore, we analyzed the activity of DAPK upon prolonged mitotic arrest induced by Plk1 depletion. In Plk1-MM siRNA -treated, exponentially growing cells, the DAPK/pS308 signal was below the limit of detection (Figure 1E, lane 2 and Supplementary information, Figure S1D, lane 1). In contrast, at 24 $h$ following transfection with Plk1 siRNA, the DAPK/ pS308 signal was clearly elevated (Figure 1E, lane 3 and Supplementary information, Figure S1D, lane 2), correlating with an enrichment of mitotic cells (Figure 1E, right panel and Supplementary information, Figure S1D, lower panels). The inhibitory autophosphorylation signal was markedly decreased or completely gone at $72 \mathrm{~h}$ (Figure 1E, lanes 4, 5 and Supplementary information, Figure S1D, lanes 3, 4). With a loss in DAPK/pS308 signal intensity (Figure 1E, Supplementary information, Figure S1D), the kinase activity of immunoprecipitated DAPK towards the DAPK substrate myosin light chain (MLC) was markedly increased (Figure 1F, left panel and Supplementary information, Figure S1E, upper panels). In addition, the phosphorylation status of a DAPK substrate, CAMKK2, was analyzed by western blotting us- ing the CAMKK2/pS511 antibody (Figure 1E, left panel, Supplementary information, Figure S1D, upper panels). This experiment confirmed that with decreasing levels of the DAPK/pS308 signal, the activity of DAPK towards its substrates increased, suggesting that during prolonged mitotic arrest a switch of DAPK function occurs from its autophosphorylated, inhibited form to the deathpromoting active form of DAPK. During the observation period $(0-72 \mathrm{~h})$ a robust increase in apoptotic activity was detected (Figure 1F, right panel and Supplementary information, Figure S1E, lower panels). Next we addressed whether activation of DAPK contributes to cell death induced by Plk1 inhibition. In different cell lines, cell apoptosis induced by Plk1 silencing or BI2536-mediated Plk1 inhibition could be inhibited by co-depletion of DAPK (Figure 1G, Supplementary information, Figure S1F-S1G), suggesting that DAPK is a critical component of the cell death machinery that is activated by Plk1 inhibition.

Considering that during prolonged mitotic arrest induced by Plk1 depletion, a switch of DAPK function occurs from its autophosphorylated, inhibited form to the death-promoting, active form of DAPK, we investigated DAPK activity in BI2536-treated cells. While the $\mathrm{DAPK} / \mathrm{pS} 308$ signal intensity is below the limit of detection in asynchronously growing cells, the treatment with increasing concentrations of BI2536 for $24 \mathrm{~h}$ induced a prominent autophosphorylation signal (DAPK/pS308)

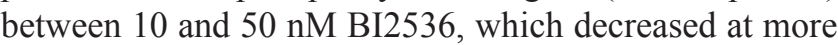
elevated drug concentrations (Figure 1H, Supplementary information, Figure S1H and S1J). While in asynchronously growing cells, DAPK activity was not detectable, treatment with low concentrations of BI2536 (10-100 $\mathrm{nM}$ ) induced increasing DAPK activity towards CAM- 
KK2 and GST-MLC (Figure 1H, 1I and Supplementary information, Figure S1H-S1J). In contrast to the use of Plk1 siRNA, which induced persistent DAPK activity towards GST-MLC (Figure 1F), elevated concentrations of BI2536 induced descending DAPK kinase activity (Figure 1I and Supplementary information, Figure S1I), suggesting that elevated concentrations of BI2536 inhibit DAPK activity, thereby preventing the functional switch to the pro-apoptotic form of DAPK. It is worth to note that higher doses are required for the inhibition of both Plk1 and DAPK kinases in in vitro kinase assays using kinases immunoprecipitated from cell lysates (Figure 1I) compared to assays using their highly purified counterparts (Figure 1B).

To investigate the effects of BI2536 treatment on DAPK activity during mitosis, we treated cells with nocodazole alone or with a combination of nocodazole and the proteasome inhibitor MG132 to trap cells in mitosis and co-treated mitotic-shake off cells with increasing BI2536 concentrations. The downregulation of the DAPK/pS308 signal in cells trapped in mitosis with stable levels of mitotic markers (Plk1, pH3) confirmed that BI2536 inhibits DAPK activity in mitotic cells and downregulation of the DAPK/pS308 is not a result of mitotic exit (Figure 1J and Supplementary information, Figure S1K). Furthermore, we analyzed the activity of DAPK upon prolonged BI2536 treatment. While the DAPK/ pS308 signal was elevated at concentrations of 10-100 $\mathrm{nM}$, but was less intense at $1000 \mathrm{nM}$ after $24 \mathrm{~h}$ treatment, the DAPK/pS308 signal was not detectable at 48 $\mathrm{h}$ and $72 \mathrm{~h}$, which might be due to the increasing number of dead cells (Supplementary information, Figure S1L). Nevertheless, we cannot rule out that DAPK undergoes de-phosphorylation during prolonged BI2536-induced arrest, which resembles the effects of Plk1-specific RNAi at $72 \mathrm{~h}$ (Figure 1E and Supplementary information, S1D).

To analyze DAPK activity in response to other antimitotic drugs, we used another Plk1 inhibitor, called BI6727. While the DAPK/pS308 signal intensity was below the limit of detection in asynchronously growing HeLa cells, the treatment with increasing BI6727 concentrations (10-100 nM) for $24 \mathrm{~h}$ induced a DAPK/pS308 signal (Supplementary information, Figure S1M). Surprisingly, this signal decreased at more elevated concentrations (Supplementary information, Figure S1M). Since BI6727 is structurally related to BI2536, we tested its impact on the activity of DAPK using in vitro kinase assays. Compared to BI2536, it was less potent, but still had the ability to suppress DAPK activity partially (Supplementary information, Figure S1N), which could account for the observation of reduced DAPK/pS308 signals at high BI6727 doses (Supplementary information, Figure S1M).
Direct binding of BI6727 to DAPK was demonstrated with the TNP-ATP displacement assay (Supplementary information, Figure S1O). Nevertheless, BI6727 showed a lower affinity to DAPK compared to BI2536 (Supplementary information, Figure S1O).

Taken together, the present study revealed novel targets of the clinical inhibitor BI2536. We present a previously unknown mitotic regulation of DAPK and its pro-apoptotic activation upon mitotic arrest induced by the Plk1 inhibitors BI2536 and BI6727. A clinical trial using BI6727 for the treatment of patients with solid tumors showed maximum plasma concentrations between 30-2 $340 \mathrm{nM}$ depending on the dose given [14], indicating that the concentrations used in our study are clinically relevant. Thus, a prerequisite for the design of an optimized cancer therapy is to better understand the kinase selectivity of novel inhibitors. Careful dose-finding studies in cancer trials targeting Plk1 are necessary to prevent the inhibition of DAP kinases, which would counteract the pro-apoptotic effect of Plk1 inhibition.

\section{Acknowledgments}

This work was supported by grants from the German Cancer Consortium (DKTK, Heidelberg), Carls-Stiftung, Deutsche Krebshilfe and BANSS Stiftung.

\section{Monika Raab ${ }^{1,3}$, Fiona Pachl' ${ }^{2}$, Andrea Krämer ${ }^{1}$, Elisabeth Kurunci-Csacsko ${ }^{1}$, Christina Dötsch ${ }^{1}$, Rainald Knecht ${ }^{3}$, Sven Becker ${ }^{1}$, Bernhard Kuster ${ }^{2,4}$, Klaus Strebhardt ${ }^{1,4}$}

\footnotetext{
${ }^{I}$ Department of Gynecology, School of Medicine, Goethe University, Theodor-Stern-Kai 7, 60590 Frankfurt, Germany; ${ }^{2}$ Technische Universität München, Emil Erlenmeyer Forum 5, 85354 Freising, Germany; ${ }^{3}$ Head and Neck Center, UKE Hamburg, Martinistr. 52, 20246 Hamburg, Germany;

${ }^{4}$ German Cancer Consortium (DKTK), Heidelberg, Germany

Correspondence: Klaus Strebhardt

Tel: +49-69-6301 6894; Fax: +49-69-6301 6364

E-mail: strebhardt@em.uni-frankfurt.de
}

\section{References}

1 Spankuch-Schmitt B, Bereiter-Hahn J, Kaufmann M, et al. J Natl Cancer Inst 2002; 94:1863-1877.

2 Liu X, Erikson RL. Proc Natl Acad Sci USA 2003; 100:5789-5794.

3 Raab M, Kappel S, Kramer A, et al. Nat Commun 2011; 2:395.

4 Yun SM, Moulaei T, Lim D, et al. Nat Struct Mol Biol 2009; 16:876882

5 Strebhardt K. Nat Rev Drug Discov 2010; 9:643-660.

6 Strebhardt K, Ullrich A. Nat Rev Cancer 2006; 6:321-330.

7 Bantscheff M, Eberhard D, Abraham Y, et al. Nat Biotechnol 2007; 25:1035-1044.

8 Patel VJ, Thalassinos K, Slade SE, et al. J Proteome Res 2009; 8:37523759.

9 Bialik S, Kimchi A. Annu Rev Biochem 2006; 75:189-210.

10 Vas M, Merli A, Rossi GL. Biochem J 1994; 301:885-891. 
11 Bilwes AM, Quezada CM, Croal LR, et al. Nat Struct Biol 2001; 8:353-360.

12 Shohat G, Spivak-Kroizman T, Cohen O, et al. J Biol Chem 2001; 276:47460-47467.

13 Cohen O, Feinstein E, Kimchi A. EMBO J 1997; 16:998-1008.

14 Schoffski P, Awada A, Dumez H, et al. Eur J Cancer 2012; 48:179186.
(Supplementary information is linked to the online version of the paper on the Cell Research website.)

(c) (1) (5) (2) This work is licensed under the Creative Commons Attribution-NonCommercial-ShareAlike 3.0 Unported License. To view a copy of this license, visit http://creativecommons.org/licenses/by-nc-sa/3.0 